\title{
VALUES OF DEDEKIND SUMS FOR FUNCTION FIELDS
}

\author{
YOSHINORI HAMAHATA
}

Abstract: H. Rademacher and E. Grosswald raised the following questions:

1. Is $\left\{(a / c, d(a, c)) \mid a / c \in \mathbb{Q}^{*}\right\}$ dense in $\mathbb{R}^{2}$ ?

2. Is $\left\{d(a, c) \mid a / c \in \mathbb{Q}^{*}\right\}$ dense in $\mathbb{R}$ ?

D. Hickerson answered them affirmatively, and H. Ito obtained a result similar to Hickerson's for the elliptic Dedekind sums defined by R. Sczech. We consider the values of the Dedekind sum attached to a given $A$-lattice in rational function fields. The objective of this paper is to establish a result similar to those of Hickerson and Ito.

Keywords: Dedekind sums, Drinfeld modules, function fields.

\section{Introduction}

The classical Dedekind sum is defined by

$$
d(a, c)=\frac{1}{4 c} \sum_{k=1}^{c-1} \cot \left(\frac{\pi a k}{c}\right) \cot \left(\frac{\pi k}{c}\right)
$$

for coprime integers $c>0$ and $a$. For coprime positive integers $a, c$, we have the reciprocity law

$$
d(a, c)+d(c, a)=\frac{1}{12}\left(\frac{a}{c}+\frac{c}{a}+\frac{1}{a c}-3\right) .
$$

Rademacher and Grosswald [7] raised the following questions:

1. Is $\left\{(a / c, d(a, c)) \mid a / c \in \mathbb{Q}^{*}\right\}$ dense in $\mathbb{R}^{2}$ ?

2. Is $\left\{d(a, c) \mid a / c \in \mathbb{Q}^{*}\right\}$ dense in $\mathbb{R}$ ?

D. Hickerson [5] answered them affirmatively, and H. Ito [6] obtained a result similar to Hickerson's for the elliptic Dedekind sums defined by R. Sczech [8]. It is well-known that there is an analogy between a number field and a function field. In [1], we introduced the Dedekind sum attached to a given Drinfeld module.

2010 Mathematics Subject Classification: primary: 11F20; secondary: 11G09 
A rank $r$ Drinfeld module is similar to the multiplicative group $\mathbb{G}_{m}$ (resp. an elliptic curve) when $r=1$ (resp. $r \geqslant 2$ ). The Dedekind sum attached to the Carlitz module is an analog of $d(a, c)$ defined above, and the Dedekind sum attached to a Drinfeld module with rank $r \geqslant 2$ is an analog of an elliptic Dedekind sum [8]. In [4], we proved a result similar to Hickerson's for the Dedekind sum attached to the Carlitz module. The objective of the present paper is to extend our result to the Dedekind sum attached to an arbitrary Drinfeld module. We state our main results in Section 2. In Section 3, we recall some previous results from [1]. In Section 4, we prove our main results, and make some concluding remarks in Section 5 .

\section{Main results}

Before stating our result, we outline our notation and give some definitions.

Let $\mathbb{F}_{q}$ be the finite field with $q$ elements, and set $A:=\mathbb{F}_{q}[T], K:=\mathbb{F}_{q}(T)$, and $K_{\infty}:=\mathbb{F}_{q}((1 / T))$. We denote by $C_{\infty}$ the completion of an algebraic closure of $K_{\infty}$. A rank $r$-lattice $\Lambda$ in $C_{\infty}$ is a finitely generated $A$-submodule of rank $r$ in $C_{\infty}$ that is discrete in the topology of $C$. For such an $A$-lattice $\Lambda$, we define the product $e_{\Lambda}(z)=z \prod_{0 \neq \lambda \in \Lambda}(1-z / \lambda)$. The product converges uniformly on bounded sets in $C_{\infty}$, and defines a map $e_{\Lambda}: C_{\infty} \rightarrow C_{\infty}$. The map $e_{\Lambda}$ has the following properties:

(E1) $e_{\Lambda}$ is entire in the rigid analytic sense, and surjective;

(E2) $e_{\Lambda}$ is $\mathbb{F}_{q}$-linear and $\Lambda$-periodic;

(E3) $e_{\Lambda}$ has simple zeros at the points of $\Lambda$, and no other zeros;

(E4) $d e_{\Lambda}(z) / d z=e_{\Lambda}^{\prime}(z)=1$.

For every $a \in A$, there exists a unique polynomial $\phi_{a}=\phi_{a}^{\Lambda}$ of the form $\sum l_{i}\left(\phi_{a}\right) z^{q^{i}}$ such that $\phi_{a}\left(e_{\Lambda}(z)\right)=e_{\Lambda}(a z)$. Let $\tau=X^{q}$ and let $C_{\infty}\{\tau\}$ be the non-commutative ring in $\tau$ with the commutation rule $c^{q} \tau=\tau c\left(c \in C_{\infty}\right)$. There exists a unique positive integer $r$ such that for for any $a \in A \backslash\{0\}$,

$$
\phi_{a}=\sum_{i=0}^{r \operatorname{deg} a} l_{i}\left(\phi_{a}\right) \tau^{i} \quad\left(l_{0}\left(\phi_{a}\right)=a\right) .
$$

Then, the map $\phi: A \rightarrow C_{\infty}\{\tau\}, a \mapsto \phi_{a}$ is called a Drinfeld module of rank $r$ over $C_{\infty}$. Because $\phi$ is an $\mathbb{F}_{q}$-linear ring homomorphism, the values $\phi_{a}(a \in A)$ are determined by $\phi_{T}$. The rank one Drinfeld module $\rho: A \rightarrow C_{\infty}\{\tau\}$ defined by $\rho_{T}(z)=T z+z^{q}$ is called the Carlitz module. There is a one-to-one correspondence between the set of rank $r A$-lattices and the set of rank $r$ Drinfeld modules given by

$$
\phi_{a}\left(e_{\Lambda}(z)\right)=e_{\Lambda}(a z) \quad(a \in A) .
$$

Let $\Lambda$ be a rank $r A$-lattice. Using (E4), $e_{\Lambda}(z)^{-1}$ is expressed by

$$
e_{\Lambda}(z)^{-1}=\frac{1}{z}+\sum_{0 \neq \lambda \in \Lambda} \frac{1}{z-\lambda},
$$


which is similar to

$$
\cot z=\frac{1}{z}+\sum_{n=1}^{\infty}\left(\frac{1}{z+\pi n}+\frac{1}{z-\pi n}\right)
$$

and

$$
E_{1}(z)=E_{1}(z ; \mathbb{Z} \omega+\mathbb{Z})=\left.\sum_{l \in \mathbb{Z} \omega+\mathbb{Z}, l+z \neq 0}(z+l)^{-1}|z+l|^{-s}\right|_{s=0}
$$

for a lattice $\mathbb{Z} \omega+\mathbb{Z}$ in $\mathbb{C}$. This observation leads us to the definition of Dedekind sums in function fields. For coprime $a, c \in A \backslash\{0\}$, we define the Dedekind sum by

$$
s_{\Lambda}(a, c)=\frac{1}{c} \sum_{0 \neq \lambda \in \Lambda / c \Lambda} e_{\Lambda}\left(\frac{a \lambda}{c}\right)^{-1} e_{\Lambda}\left(\frac{\lambda}{c}\right)^{-1} .
$$

When $\Lambda / c \Lambda=\{0\}, s_{\Lambda}(a, c)$ is defined as zero. Using (E2), we find that $s_{\Lambda}(a, c)=0$ for $q>3$. Hence we assume that $q=3$ or 2 . Let $\phi$ be the Drinfeld module corresponding to $\Lambda$, and let $\phi_{a}=T+l_{1}\left(\phi_{a}\right) \tau+\cdots+\cdots+l_{r \operatorname{deg} a}\left(\phi_{a}\right) \tau^{r \operatorname{deg} a}$. Our first result is as follows:

Theorem 1. Assume that $q=3$ or 2 . Let $L$ be the A-lattice corresponding to the Carlitz module $\rho$. Then

$$
s_{\Lambda}(a, c)= \begin{cases}l_{1}\left(\phi_{T}\right) s_{L}(a, c) & (q=3), \\ l_{1}\left(\phi_{T}\right)^{2} s_{L}(a, c) & (q=2)\end{cases}
$$

for coprime $a, c \in A \backslash\{0\}$.

As corollaries, we have the following results:

\section{Theorem 2.}

(i) If $q=3$, then $s_{\Lambda}\left(\left[a_{0}, \ldots, a_{r}\right]\right)$ is written as

$$
\begin{cases}\frac{l_{1}\left(\phi_{T}\right)}{T^{3}-T}\left(\left[0, a_{1}, \ldots, a_{r}\right]\right. & \\ \left.+(-1)^{r+1}\left[0, a_{r}, \ldots, a_{1}\right]+a_{1}-a_{2}+\cdots+(-1)^{r+1} a_{r}\right) & (r \geqslant 1), \\ 0 & (r=0) .\end{cases}
$$

(ii) If $q=2$, then $s_{\Lambda}\left(\left[a_{0}, \ldots, a_{r}\right]\right)$ is written as

$$
\begin{cases}\frac{l_{1}\left(\phi_{T}\right)^{2}}{T^{4}+T^{2}}\left(\left[0, a_{1}, \ldots, a_{r}\right]+(-1)^{r+1}\left[0, a_{r}, \ldots, a_{1}\right]\right. & \\ \left.+\prod_{i=1}^{r}\left[0, a_{i}, \ldots, a_{r}\right]+a_{1}-a_{2}+\cdots+(-1)^{r+1} a_{r}+r-1\right) & (r \geqslant 1), \\ 0 & (r=0) .\end{cases}
$$

Here $\left[a_{0}, a_{1}, \ldots, a_{n}\right]$ is a continued fraction defined by (4.2) in Section 4. 
Let us define the modified Dedekind sum $t_{\Lambda}(a, c)$ by

$$
t_{\Lambda}(a, c)= \begin{cases}s_{\Lambda}(a, c) / l_{1}\left(\phi_{T}\right) & (q=3), \\ s_{\Lambda}(a, c) / l_{1}\left(\phi_{T}\right)^{2} & (q=2) .\end{cases}
$$

We now present a density result for $t_{\Lambda}(a, c)$.

Theorem 3. Assume that $q=3$ or 2 . If $l_{1}\left(\phi_{T}\right)=0$, then $s_{\Lambda}(a, c)=0$.

If $l_{1}\left(\phi_{T}\right) \neq 0$, then

(i) $\left\{\left(a / c, t_{\Lambda}(a, c)\right) \mid a / c \in K^{*}\right\}$ is dense in $K_{\infty}^{2}$.

(ii) $\left\{t_{\Lambda}(a, c) \mid a / c \in K^{*}\right\}$ is dense in $K_{\infty}$.

Remark 4. Theorem 3 for $r \geqslant 2$ is an analog of Ito's result (Theorem 2 in [6]) for the elliptic Dedekind sum $D(h, k)$ defined by

$$
D(h, k)=\frac{1}{k} \sum_{\mu \in(\mathbb{Z} \omega+\mathbb{Z}) / k(\mathbb{Z} \omega+\mathbb{Z})} E_{1}\left(\frac{h \mu}{k}\right) E_{1}\left(\frac{\mu}{k}\right)
$$

for $h, k \in \mathfrak{O}=\{m \in \mathbb{C} \mid m(\mathbb{Z} \omega+\mathbb{Z}) \subset \mathbb{Z} \omega+\mathbb{Z}\}$ with $k \neq 0$.

\section{Review of some previous results}

Let us review the previous results obtained in [1]. Let $\Lambda$ be the $A$-lattice that corresponds to a Drinfeld module $\phi$. For $a \in A \backslash\{0\}$, let $\phi[a]$ be the set of $a$-division points defined by $\phi[a]=\left\{x \in C_{\infty} \mid \phi_{a}(x)=0\right\}$. Put

$$
E_{k}(\phi[a])=\sum_{0 \neq x \in \phi[a]} \frac{1}{x^{k}} .
$$

Then the reciprocity law for our Dedekind sums is as follows:

Theorem 5 ([1]). If $a, c \in A \backslash\{0\}$, then

$$
s_{\Lambda}(a, c)+s_{\Lambda}(c, a)=\frac{1}{a c}\left(E_{2}(\phi[a])+E_{2}(\phi[c])-E_{1}(\phi[a]) E_{1}(\phi[c])\right) .
$$

We recall that

$$
E_{k}(\phi[a])= \begin{cases}l_{1}\left(\phi_{a}\right) / a & (k=q-1), \\ 0 & (k=1, \ldots, q-2 \text { if } q>2) .\end{cases}
$$

Rewriting (3.1) with the aid of (3.2), we get the following:

Theorem 6 ([1]). Let $a, c \in A \backslash\{0\}$ be coprime.

(i) If $q=3$, then

$$
s_{\Lambda}(a, c)+s_{\Lambda}(c, a)=\frac{1}{a c}\left(\frac{l_{1}\left(\phi_{a}\right)}{a}+\frac{l_{1}\left(\phi_{c}\right)}{c}\right) .
$$

(ii) If $q=2$, then

$$
s_{\Lambda}(a, c)+s_{\Lambda}(c, a)=\frac{1}{a c}\left(\frac{l_{1}\left(\phi_{a}\right)^{2}}{a^{2}}+\frac{l_{1}\left(\phi_{c}\right)^{2}}{c^{2}}+\frac{l_{1}\left(\phi_{a}\right) l_{1}\left(\phi_{c}\right)}{a c}\right) .
$$




\section{Proof of Theorems 1, 2, and 3}

It is easy to see that Theorem 2 follows from Theorem 8 below, and that Theorem 3 follows from Theorem 9 below. Hence it suffices to prove Theorem 1.

\subsection{The case $l_{1}\left(\phi_{T}\right)=0$}

Let $a / c=\left[a_{0}, a_{1}, \ldots, a_{n}\right]$. By Theorem $6, s_{\Lambda}(a / c)=-s_{\Lambda}(c / a)$. Hence

$$
\begin{aligned}
s_{\Lambda}(a / c) & =s_{\Lambda}\left(\left[0, a_{1}, \ldots, a_{n}\right]\right)=-s_{\Lambda}\left(\left[a_{1}, a_{2}, \ldots, a_{n}\right]\right)=\cdots \\
& =(-1)^{n} s_{\lambda}\left(a_{n}\right)=0 .
\end{aligned}
$$

\subsection{The case $l_{1}\left(\phi_{T}\right) \neq 0$}

First, we recall the results of the Dedekind sum attached to the Carlitz module $\rho$ defined by $\rho_{T}=T+\tau$. If $L$ is the $A$-lattice corresponding to $\rho$, then according to Goss [3], we have

$$
l_{1}\left(\rho_{a}\right)=\frac{a^{q}-a}{T^{q}-T}
$$

for $a \in A \backslash\{0\}$. Using this, we have

Proposition 7 ([4]). Let $a, c \in A \backslash\{0\}$ be coprime.

(i) If $q=3$, then

$$
s_{L}(a, c)+s_{L}(c, a)=\frac{1}{T^{3}-T}\left(\frac{a}{c}+\frac{c}{a}+\frac{1}{a c}\right) .
$$

(ii) If $q=2$, then

$$
s_{L}(a, c)+s_{L}(c, a)=\frac{1}{T^{4}+T^{2}}\left(\frac{a}{c}+\frac{c}{a}+\frac{1}{a}+\frac{1}{c}+\frac{1}{a c}+1\right) .
$$

For $x \in K$, we define a sequence $\left(x_{n}\right)_{n \geqslant 0}$ by

$$
x_{0}=x, \quad x_{n+1}=\frac{1}{x_{n}-a_{n}},
$$

where $a_{n}$ is the polynomial part of the Laurent expansion $x_{n}=\sum_{i=-\infty}^{k} A_{i} T^{i}$. This sequence yields the unique continued fraction development of $x$ given by

$$
x=\left[a_{0}, a_{1}, \ldots, a_{n}\right]:=a_{0}+\frac{1}{a_{1}+\frac{1}{\cdots+\frac{1}{a_{n-1}+\frac{1}{a_{n}}}} .} .
$$

We write $s_{\Lambda}(a / c)=s_{\Lambda}(a, c)$ because the value $s_{\Lambda}(a, c)$ depends on $a / c$. Then $s_{\Lambda}(a / c)$ is $A$-periodic, i.e., $s_{\Lambda}(a / c+b)=s_{\Lambda}(a / c)$ for $b \in A$. For $\Lambda=L$, the value $s_{L}(a / c)$ is described by the continued fraction development of $a / c$. 


\section{Theorem 8 ([4]).}

(i) If $q=3$, then $s_{L}\left(\left[a_{0}, \ldots, a_{r}\right]\right)$ is written as

$$
\begin{cases}\frac{1}{T^{3}-T}\left(\left[0, a_{1}, \ldots, a_{r}\right]+(-1)^{r+1}\left[0, a_{r}, \ldots, a_{1}\right]\right. & \\ \left.+a_{1}-a_{2}+\cdots+(-1)^{r+1} a_{r}\right) & (r \geqslant 1), \\ 0 & (r=0) .\end{cases}
$$

(ii) If $q=2$, then $s_{L}\left(\left[a_{0}, \ldots, a_{r}\right]\right)$ is written as

$$
\begin{cases}\frac{1}{T^{4}+T^{2}}\left(\left[0, a_{1}, \ldots, a_{r}\right]+(-1)^{r+1}\left[0, a_{r}, \ldots, a_{1}\right]\right. & \\ \left.+\prod_{i=1}^{r}\left[0, a_{i}, \ldots, a_{r}\right]+a_{1}-a_{2}+\cdots+(-1)^{r+1} a_{r}+r-1\right) & (r \geqslant 1), \\ 0 & (r=0) .\end{cases}
$$

It should be noted that the value $s_{L}(a / c)$ is uniquely determined from the reciprocity law in Proposition 7. From this theorem, we have

Theorem 9 ([4]). Assume that $q=3$ or 2.

(i) $\left\{\left(a / c, s_{L}(a, c)\right) \mid a / c \in K^{*}\right\}$ is dense in $K_{\infty}^{2}$.

(ii) $\left\{s_{L}(a, c) \mid a / c \in K^{*}\right\}$ is dense in $K_{\infty}$.

We next extend Theorem 9 to the case $l_{1}\left(\phi_{T}\right)=1$. Let $\phi_{a}=a+l_{1}\left(\phi_{a}\right) \tau+$ $\cdots+l_{r \operatorname{deg} a}\left(\phi_{a}\right) \tau^{r \operatorname{deg} a}$ for $a \in A$. Because $\phi_{T}$ is expressed as

$$
\phi_{T}=\rho_{T}+l_{2}\left(\phi_{T}\right) \tau^{2}+\cdots+l_{r}\left(\phi_{T}\right) \tau^{r},
$$

the coefficient of $\tau$ in $\phi_{a}$ coincides with that of $\tau$ in $\rho_{a}$. Hence $l_{1}\left(\phi_{a}\right)=$ $\left(a^{q}-a\right) /\left(T^{q}-T\right)$. Using Theorem $6, s_{\Lambda}(a, c)$ has the same reciprocity law as that for $s_{L}(a, c)$. As mentioned in 4.2.1, the value $s_{L}(a, c)$ is uniquely determined by the reciprocity law. Therefore it follows that $s_{\Lambda}(a, c)=s_{L}(a, c)$.

Finally, we consider the general case $l_{1}\left(\phi_{T}\right) \neq 0$. Let $\phi_{a}=a+l_{1}\left(\phi_{a}\right) \tau+$ $\cdots+l_{r \operatorname{deg} a}\left(\phi_{a}\right) \tau^{r \operatorname{deg} a}$ for $a \in A$. We take $\gamma \in C_{\infty}$ such that $l_{1}\left(\phi_{T}\right)=\gamma^{q-1}$. Define a Drinfeld module $\psi$ by $\psi_{T}=\gamma \phi_{T} \gamma^{-1}$, and writing $\psi_{a}=a+l_{1}\left(\psi_{a}\right) \tau+$ $\cdots+l_{r \operatorname{deg} a}\left(\psi_{a}\right) \tau^{r \operatorname{deg} a}$ for $a \in A$, we see that $l_{1}\left(\psi_{T}\right)=1$. Hence $l_{1}\left(\psi_{a}\right)=$ $\left(a^{q}-a\right) /\left(T^{q}-T\right)$. We have

$$
l_{1}\left(\phi_{a}\right)=\gamma^{q-1} l_{1}\left(\psi_{a}\right)=l_{1}\left(\phi_{T}\right) \frac{a^{q}-a}{T^{q}-T} .
$$

Let

$$
t_{\Lambda}(a, c)= \begin{cases}s_{\Lambda}(a, c) / l_{1}\left(\phi_{T}\right) & (q=3), \\ s_{\Lambda}(a, c) / l_{1}\left(\phi_{T}\right)^{2} & (q=2) .\end{cases}
$$

Then by Theorem 6 , it holds that

$$
t_{\Lambda}(a, c)+t_{\Lambda}(c, a)= \begin{cases}\frac{1}{T^{3}-T}\left(\frac{a}{c}+\frac{c}{a}+\frac{1}{a c}\right) & (q=3), \\ \frac{1}{T^{4}+T^{2}}\left(\frac{a}{c}+\frac{c}{a}+\frac{1}{a}+\frac{1}{c}+\frac{1}{a c}+1\right) & (q=2) .\end{cases}
$$

This is just the reciprocity law for $s_{L}(a, c)$ in Proposition 7. Thus we conclude that $t_{\Lambda}(a, c)=s_{L}(a, c)$, which completes the proof of Theorem 1 . 


\section{Concluding remarks}

As an application of Theorem 1, we make some remarks on modular forms for $G L(r, A)$. Suppose $r \geqslant 2$. Set

$$
\Omega^{r}=\mathbb{P}^{r-1}\left(C_{\infty}\right) \backslash\left\{K_{\infty} \text {-rational hyperplanes }\right\} .
$$

For $\underline{\omega}=\left(\omega_{1}: \cdots: \omega_{r}\right) \in \Omega^{r}$, put $z_{i}=\omega_{i} / \omega_{r}(i=1, \ldots, r)$ and $\Lambda_{\underline{\omega}}=A z_{1}+$ $\cdots+A z_{r}$. We write $\phi \underline{\underline{\omega}}$ for the Drinfeld module corresponding to $\bar{\Lambda}_{\underline{\omega}}$, and let $\phi \frac{\omega}{T}=T+l_{1}(\underline{\omega}) \tau+\cdots+l_{r}(\underline{\omega}) \tau^{r}$. The coefficient $l_{i}(\underline{\omega})$ is a modular form of weight $q^{i}-1$ for $G L(r, A)$ in the sense of Gekeler [2]. Theorem 1 yields the following result:

Theorem 10. Assume that $q=3$ or 2 . Let $L$ be the A-lattice correspnding to the Carlitz module $\rho$. Then for coprime $a, c \in A \backslash\{0\}, s_{\Lambda_{\underline{\omega}}}(a, c)$ is a modular form of weight $q-1$ for $G L(r, A)$, and is written as

$$
s_{\Lambda_{\underline{\omega}}}(a, c)= \begin{cases}s_{L}(a, c) l_{1}(\underline{\omega}) & (q=3), \\ s_{L}(a, c) l_{1}(\underline{\omega})^{2} & (q=2) .\end{cases}
$$

\section{References}

[1] A. Bayad and Y. Hamahata, Higher dimensional Dedekind sums in function fields, Acta Arithmetica 152 (2012), 71-80.

[2] E.-U. Gekeler, Drinfeld modules and local fields of positive characteristic, in: Geometry \& Topology Monographs 3: Invitation to higher local fields Part II, pp.239-253.

[3] D. Goss, Basic Structures of Function Field Arithmetic, Springer, 1998.

[4] Y. Hamahata, Continued fractions and Dedekind sums for function fields, in: Number Theory and Related Fields, pp. 187-197, Springer Proc. Math. Stat. 43, Springer, New York, 2013.

[5] D. Hickerson, Continued fractions and density results for Dedekind sums, J. Reine Angew. Math. 290 (1977), 113-116.

[6] H. Ito, A density result for elliptic Dedekind sums, Acta Arithmetica 112 (2004), 199-208.

[7] H. Rademacher and E. Grosswald, Dedekind Sums, The Mathematical Association of America, Washington, D.C., 1972.

[8] R. Sczech, Dedekindsummen mit elliptischen Functionen, Invent. Math. 76 (1984), 523-551.

Address: Yoshinori Hamahata: Department of Applied Mathematics, Okayama University of Science, Ridai-cho 1-1, Okayama 700-0005, Japan.

E-mail: hamahata@xmath.ous.ac.jp

Received: 17 April 2013 